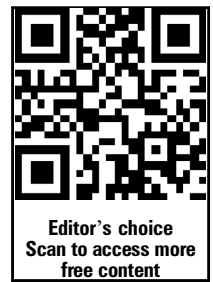

free content

- Additional material is published online only. To view please visit the journal online (http://dx.doi.org/10.1136/ gutjnl-2013-305964).

1 Department of Surgery, Vanderbilt University School of Medicine, Nashville, Tennessee, USA

${ }^{2}$ Epithelial Biology Center, Vanderbilt University School of Medicine, Nashville, Tennessee, USA

${ }^{3}$ Department of Pathology, Vanderbilt University School of Medicine, Nashville, Tennessee, USA

${ }^{4}$ Severance Biomedical Science Institute, Yonsei University College of Medicine, Seoul, Korea

${ }^{5}$ Nashville VA Medical Center, Vanderbilt University School of Medicine, Nashville, Tennessee USA

${ }^{6}$ Department of Cell and Developmental Biology, Vanderbilt University School of Medicine, Nashville, Tennessee, USA

\section{Correspondence to} Dr J R Goldenring, Section of Surgical Sciences, Vanderbilt University School of Medicine, Epithelial Biology Center, 10435G MRB-IV

2213 Garland Avenue, Nashville, TN 37232-2733, USA:

jim.goldenring@vanderbilt.edu

EC and JTR contributed equally.

Received 27 August 2013 Revised 5 December 2013 Accepted 3 January 2014 Published Online First 31 January 2014

\section{CrossMark}

To cite: Choi E, Roland JT, Barlow BJ, et al. Gut 2014;63:1711-1720.

\title{
Cell lineage distribution atlas of the human stomach reveals heterogeneous gland populations in the gastric antrum
}

\author{
Eunyoung Choi, ${ }^{1,2}$ Joseph T Roland, ${ }^{1,2}$ Brittney J Barlow, ${ }^{1,2}$ Ryan O'Neal, ${ }^{1,2}$ \\ Amy E Rich, ${ }^{2,3}$ Ki Taek Nam, ${ }^{1,2,4}$ Chanjuan Shi, ${ }^{3}$ James R Goldenring ${ }^{1,2,5,6}$
}

\section{ABSTRACT}

Objective The glands of the stomach body and antral mucosa contain a complex compendium of cell lineages. In lower mammals, the distribution of oxyntic glands and antral glands define the anatomical regions within the stomach. We examined in detail the distribution of the full range of cell lineages within the human stomach.

Design We determined the distribution of gastric gland cell lineages with specific immunocytochemical markers in entire stomach specimens from three non-obese organ donors.

Results The anatomical body and antrum of the human stomach were defined by the presence of ghrelin and gastrin cells, respectively. Concentrations of somatostatin cells were observed in the proximal stomach. Parietal cells were seen in all glands of the body of the stomach as well as in over $50 \%$ of antral glands. MIST1 expressing chief cells were predominantly observed in the body although individual glands of the antrum also showed MIST1 expressing chief cells. While classically described antral glands were observed with gastrin cells and deep antral mucous cells without any parietal cells, we also observed a substantial population of mixed type glands containing both parietal cells and $\mathrm{G}$ cells throughout the antrum.

Conclusions Enteroendocrine cells show distinct patterns of localisation in the human stomach. The existence of antral glands with mixed cell lineages indicates that human antral glands may be functionally chimeric with glands assembled from multiple distinct stem cell populations.

\section{INTRODUCTION}

Among gastrointestinal tissues, the gastric mucosa is constructed from a more complex set of short lived and long lived cell lineages. ${ }^{1-5}$ While studies over the past 20 years have detailed the origin and distribution of cell lineages in the rodent stomach, ${ }^{1-5}$ far fewer studies have addressed the distribution of lineages in the human stomach. The rodent stomach is divided into three discrete anatomical zones, including the squamous lined forestomach, the glandular-oxyntic (body) region containing acid secreting parietal cells and pepsinogen secreting chief cells, and the antrum containing gastrin cells. ${ }^{6}$ In all rodents studied as well as other mammalian species, the border between the antrum and the body contains areas of transitional glands with fewer parietal cells before the initiation of antral mucous glands that lack parietal cells. ${ }^{6}{ }^{7}$ It is thought that the

\section{Significance of this study}

What is already known on this subject?

- Enteroendocrine cell lineages are distributed throughout the human stomach and regulate secretory physiology.

- In the human stomach, parietal cell containing oxyntic glands are found in the body of the stomach.

- In the human stomach, gastrin cell containing mucous glands are found in the antrum.

What are the new findings?

- Enteroendocrine cells are regionally concentrated within the human stomach.

- Human antrum contains three types of glands: oxyntic, antral and mixed.

- The presence of both gastrin cells and parietal cells in mixed glands suggests that multiple stem cells may reside in human antral glands.

How might it impact on clinical practice in the foreseeable future?

- The presence of antral oxyntic and mixed glands may suggest that specific pathologies, such as intestinal metaplasia, in the antrum could arise from these glands.

boundaries between these regions are dictated by the developmental expression of specific transcription factors, especially sex determining region Y-box 2 (SOX2) and pancreatic and duodenal homeobox 1 $(\mathrm{Pdx} 1) .{ }^{8} 9$ Thus Pdx1 expression defines the region of rodent antrum and deletion of Pdx1 causes failure of antrum formation. ${ }^{10}$

In contrast with mice, the geographic anatomy of the human stomach is far less detailed. The human stomach does not have a squamous forestomach region but rather is divided anatomically into three regions: a proximal peri-oesophageal cardia, the glandular body and the antrum. ${ }^{11}$ Traditionally, these regions have been grossly defined by the positions of the nerves of Latrajet. While much of the present literature suggests that human gastric lineages are distributed in a manner similar to that in rodents and other animals, few studies have previously defined in detail the distribution of cell types within the human stomach. 
We have now evaluated the geographic distribution of cell lineages within the human stomach by quantitative determination of cell numbers throughout the gastric mucosa from nonobese organ donors. These results have revealed that cell lineages are not uniformly distributed throughout the stomach and that there are distinct and important differences in humans compared with other species. Enteroendocrine cell lineages were concentrated in discrete regions within the proximal and distal stomach. Ghrelin and gastrin best defined the anatomical body and antrum, respectively. Importantly, parietal cells were distributed in glands throughout the human antrum. Indeed, the human antrum appears to contain three distinct types of glands containing (1) parietal and chief cells (oxyntic-type glands), (2) gastrin and trefoil factor 2 (TFF2) positive mucous cells (antraltype glands) as well as (3) both parietal cells and gastrin cells (mixed-type glands). The results suggest that the human stomach has a unique geographical distribution of lineages with acid secreting cells throughout the antrum. These findings suggest that lineage derivation in the human stomach may not follow the same rules as in mouse stomach.

\section{METHODS}

See online supplementary methods.

\section{RESULTS}

Mapping the geographic distribution of lineages in the human stomach

To map the distribution of cell lineages within the human stomach, the entire stomach was obtained from three organ donors (see online supplementary table S1). The stomachs were opened along the greater curvature and fixed overnight in formalin. The specimens were then divided into regions of $0.5 \mathrm{~cm}$ height $\times 2 \mathrm{~cm}$ wide (figure 1 ). Each region was separately embedded in paraffin with the specimen oriented to display glands along their length. Cores from these blocks $(1 \mathrm{~mm})$ were then

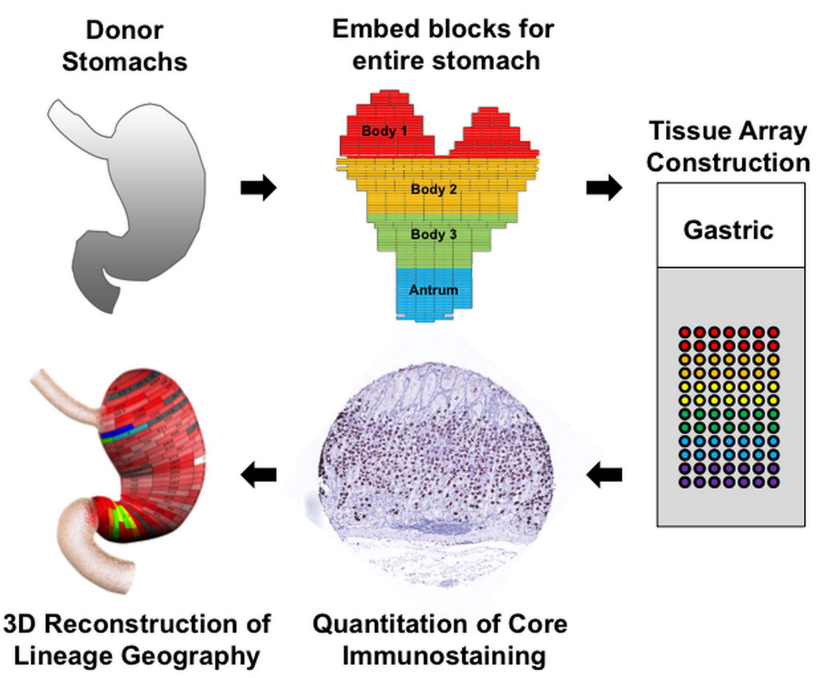

Figure 1 Procedure for geographic mapping of the cell lineages within the human stomach. The three entire donor stomachs were divided into regions of $0.5 \mathrm{~cm}$ height $\times 2 \mathrm{~cm}$ wide to embed in paraffin blocks, and $1 \mathrm{~mm}$ cores from the paraffin blocks were excised and assembled into tissue arrays. These arrays were stained with cell lineage specific antibodies, and then the cell numbers per core were determined using a digital quantitation system (Ariol SL-50). Finally, the distribution of cell lineages was displayed in three-dimensional (3D) projections of the stomach. excised and arrayed into tissue arrays that together covered the entire stomachs from the three specimens. We then stained these arrays with antibodies against specific markers of cell lineages (see online supplementary table S2). The tissue arrays were analysed by digital quantitation using an Ariol SL-50 system (Leica) and lineage abundance was quantified as cells per $1 \mathrm{~mm}$ core (figure 1). The cell numbers were then displayed in two dimensions on maps of the stomach specimen (see online supplementary figures S1-S3), and heat map colouration was developed to display the data. Finally, the two-dimensional maps were rendered onto three-dimensional projections of the stomach to display the distribution of lineages throughout the stomachs. Data for all three of the donor stomachs are shown in the online supplementary materials as two-dimensional maps (see online supplementary figures S1-S3). Three-dimensional rendering was performed only for donor No 2 stomach as a representative of our findings (figures 2 and 3). Dynamic rotating reconstructions of the three-dimensional renderings are included in the online supplementary videos 1-9. To analyse the distribution of lineages in the stomachs, each specimen was divided into three body zones and antrum (see figure 1) and the distributions of lineages were analysed as a per cent of total labelled cells (figure 4).

\section{Distribution of enteroendocrine cells in the human stomach} Traditionally, the gastric regions of the human stomach have been divided into body versus antral regions. We therefore examined the distribution of enteroendocrine cell lineages in the glands of these regions. As noted in numerous previous investigations, ${ }^{12-15}$ gastrin expressing cells were confined to the distal stomach (figures $2 \mathrm{~A}$ and $4 \mathrm{~A}$ ). In contrast, also as previously reported, ${ }^{16}$ ghrelin expressing cells were essentially excluded from the distal stomach and were abundant in the stomach body regions with a relatively even distribution throughout the body mucosa (figures $2 \mathrm{~B}$ and $4 \mathrm{~B}$ ). A similar staining pattern was also observed for obestatin, a splice variant of the ghrelin gene (data not shown). ${ }^{17}$ This inverse relationship between gastrin and ghrelin expression was observed in all three of the donor stomachs (see online supplementary figure S1 and figure 4A,B). These findings suggested that gastrin and ghrelin cells define the anatomical division between the human stomach body and antrum, respectively.

Given the asymmetric distribution of ghrelin and gastrin cells, we evaluated the distribution of other cell lineages within the three zones of the gastric body and the fourth zone of the antrum. These distributions were evaluated across all three organ donor stomach specimens (figure 4). We first examined the distribution of other enteroendocrine cells in the stomach. Chromogranin A is prominently expressed in most amine secreting enteroendocrine cells, including the histamine secreting enterochromaffin-like (ECL) cells. ${ }^{18}{ }^{19}$ Figures 2C and 4C demonstrate that chromogranin A positive cells were numerous and uniformly distributed throughout the anatomical body of the stomach defined by the ghrelin expressing cells. Significantly lower numbers of chromogranin A immunoreactive cells were also seen in the anatomical antrum. We next determined the distribution of serotonin expressing enterochromaffin cells in the stomach. Serotonin cells were far less numerous throughout the stomach, usually fewer than 10 cells per core. Compared with other markers, there was more variability in both serotonin cell numbers and distribution. Similar to findings in a previous report, ${ }^{20}$ all three donor stomachs showed a concentration of serotonin cells in the antrum (figures 2D and 4D). However, we 
A Gastrin
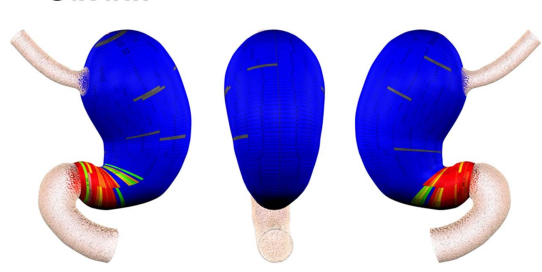

B Ghrelin
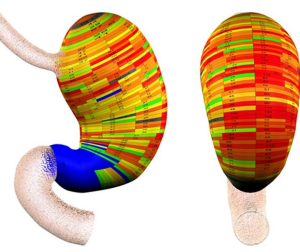

C Chromogranin A
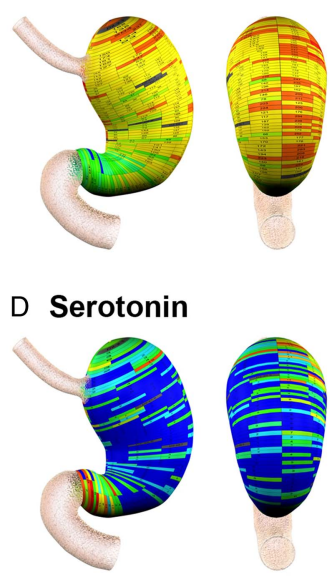

\section{E Somatostatin}

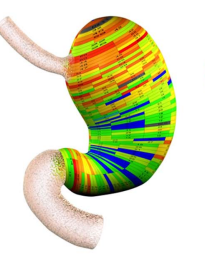

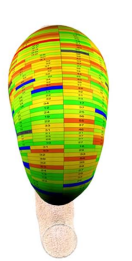
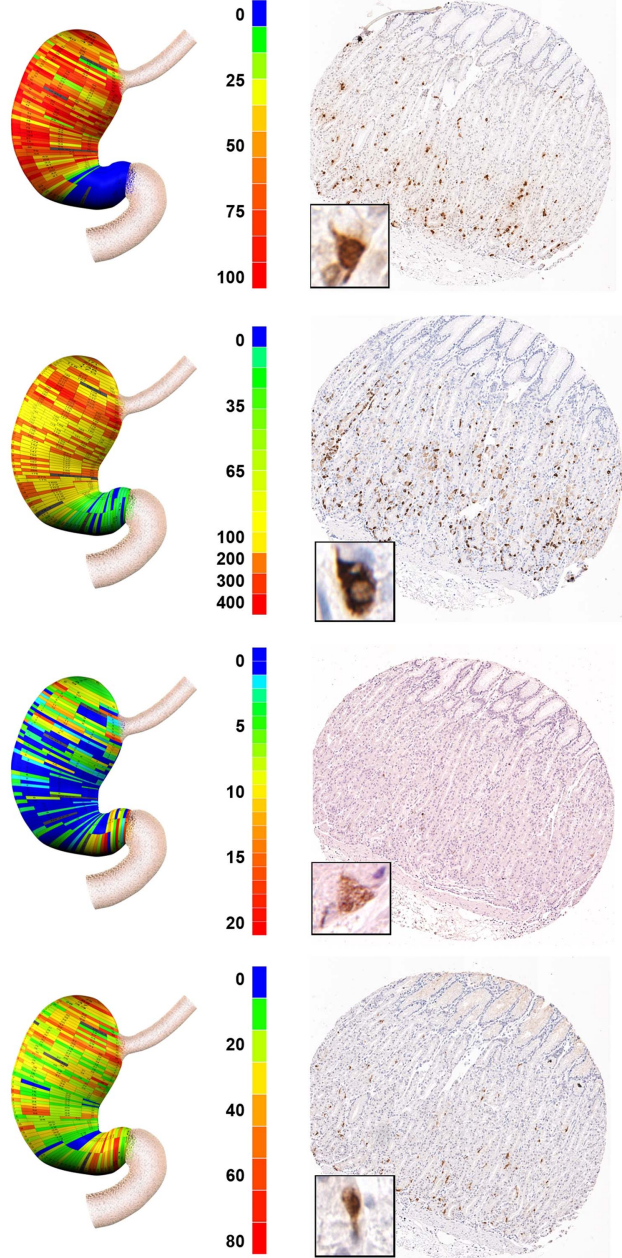

BODY
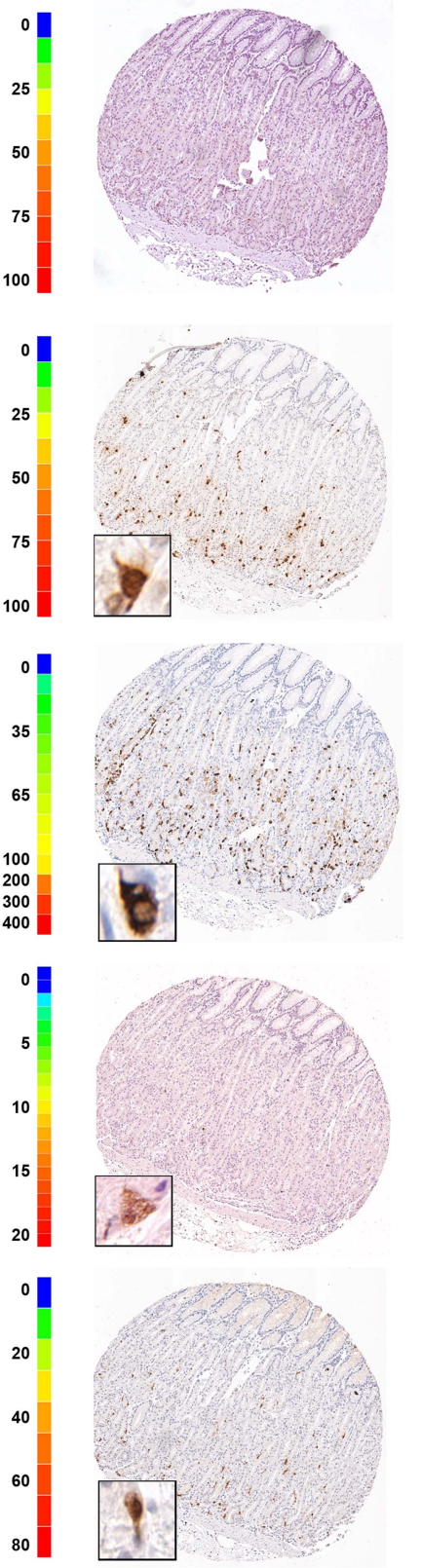
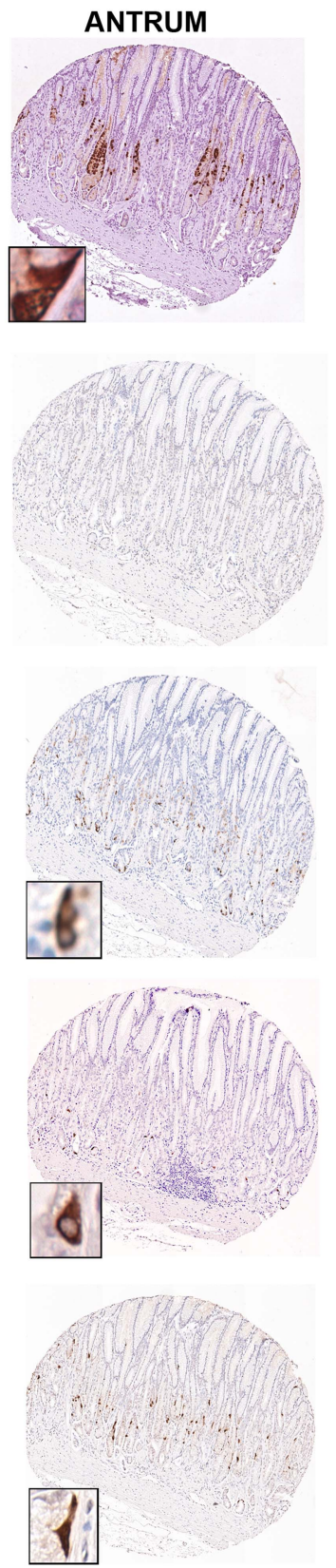

Figure 2 Geographic mapping of endocrine cell lineages. Tissue array sections from donor No 2 were stained for (A) gastrin (G cells), (B) ghrelin (X cells), (C) chromogranin A (enteroendocrine cells), (D) serotonin (enterochromaffin cells) and (E) somatostatin (D cells). Quantitated cell lineage numbers per core were mapped onto three-dimensional stomach maps to demonstrate the distribution of cells in the stomach (left panels). The scale bars represent the range of positive cells in a core. Representative cores from the body and antrum are shown on the right of each panel with high magnification insets showing the individual cell staining pattern.

also observed a concentration of serotonin cells in the proximal stomach.

To complete the analysis of enteroendocrine cells in the human stomach, we examined the distribution of somatostatin immunoreactive D cells. D cells were distributed throughout the body and antrum (figure 2E). In all three stomachs, there were significantly higher numbers of somatostatin cells in the proximal regions of the body mucosa (figure 4E). Thus the inhibitory influences of somatostatin cells are concentrated in the proximal stomach. ${ }^{21}$

In addition to the endocrine cell lineages, we also evaluated the presence of tuft cells in the normal human stomachs. Tuft cells are sensory mucosal cell lineages that form direct synapses with interneurons in the gastric wall. ${ }^{5}$ Staining for DCLK1, a marker of tuft cells, showed staining of rare individual cells in cores in the body and antrum (see online supplementary figure S4B). The tufts cells showed the characteristic morphology with a prominent apical extension (see online supplementary figure S4B). The tuft cells were extremely rare and no more than 20 tufts cells were identified in an entire set of stomach specimen cores.

\section{Distribution of mucous and secretory lineages in the human stomach}

We next examined the distribution of cells considered components of oxyntic glands: surface mucous cells, parietal cells, 
A Ki-67

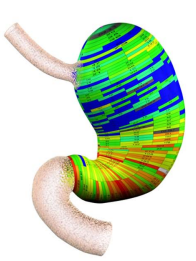

B H/K-ATPase
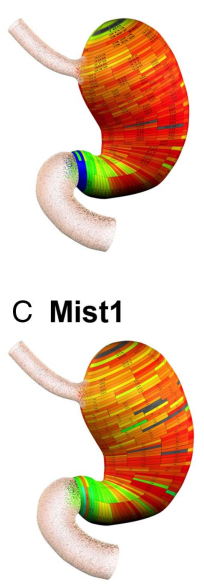

D MUC6

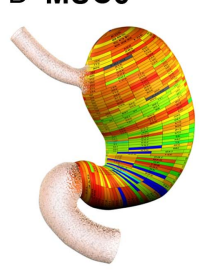

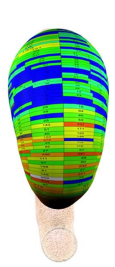
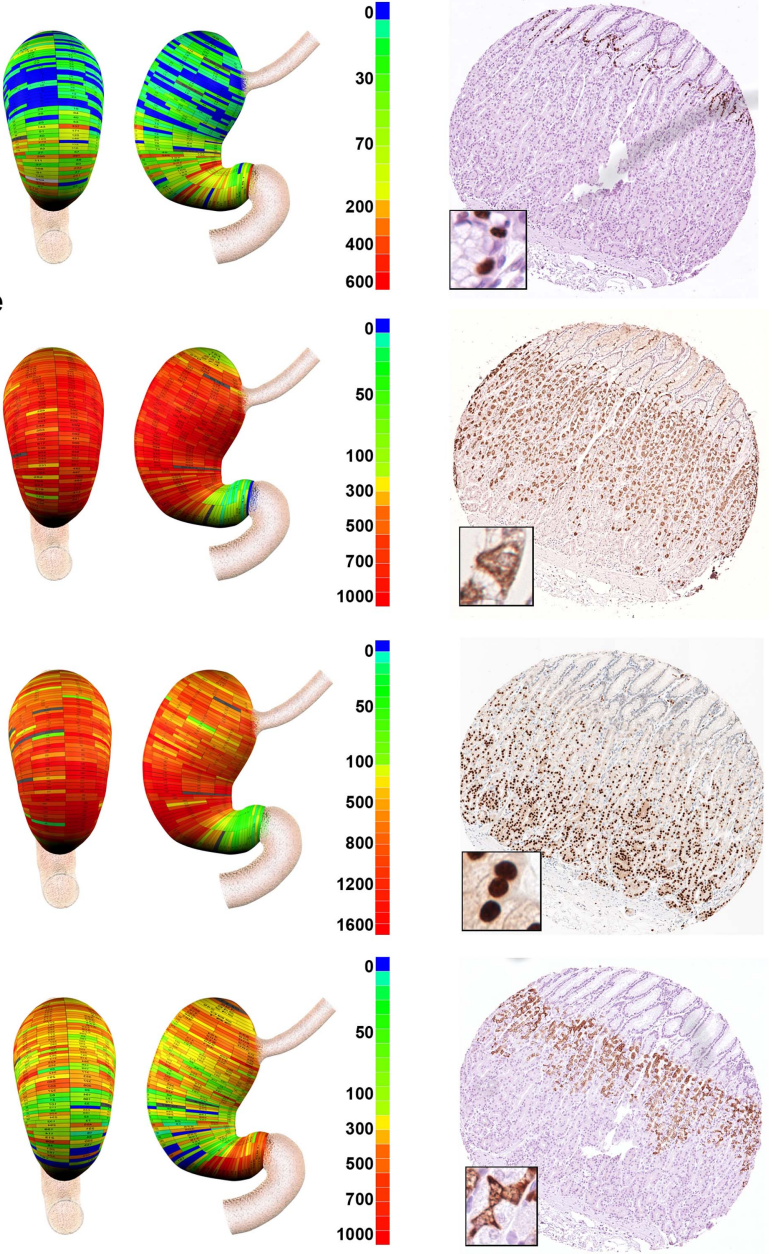
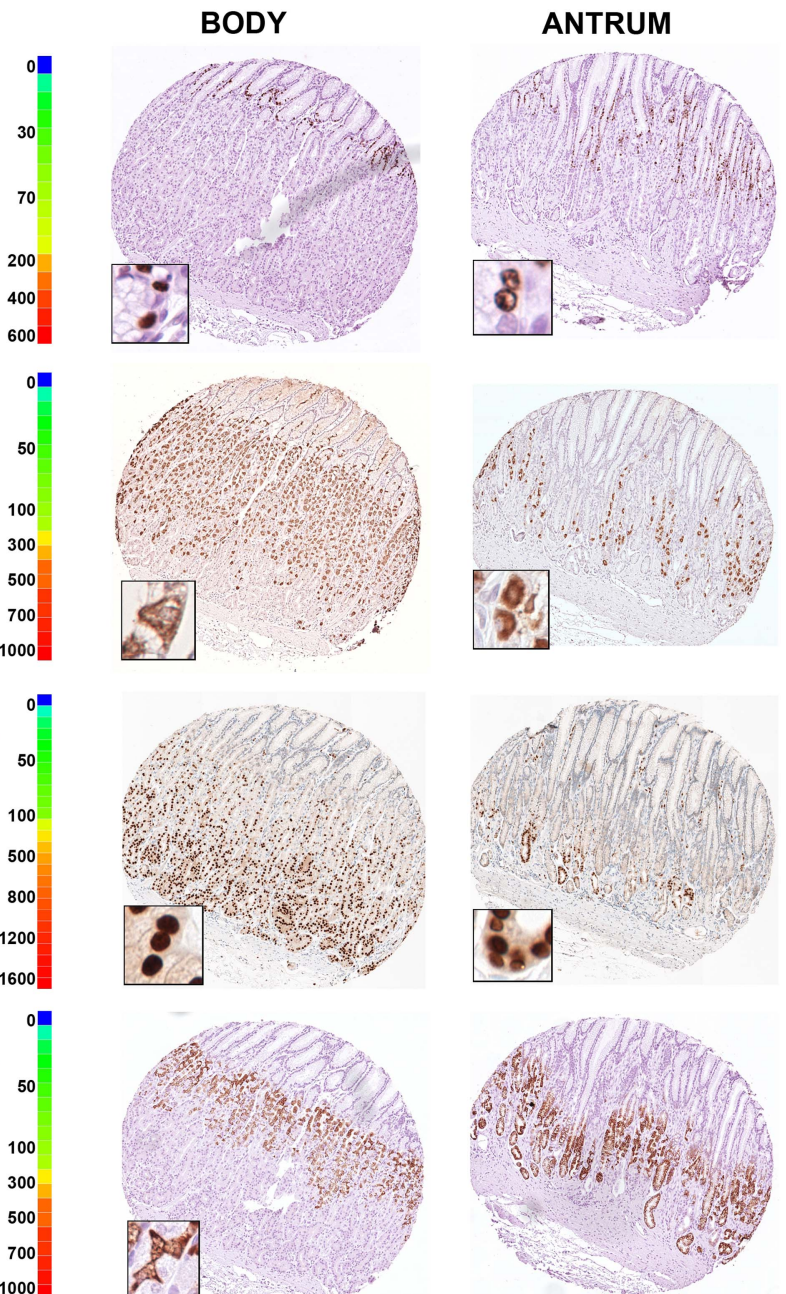

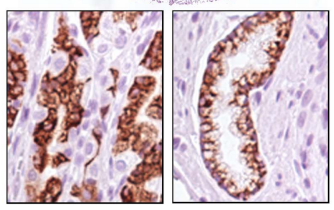

Figure 3 Geographic mapping of progenitor and oxyntic gland cell lineages. Tissue array sections were stained for (A) Ki-67 (progenitor cells), (B) H/K-ATPase (parietal cells), (C) MIST1 (chief cells) and (D) MUC6 (mucous neck cells and deep antral gland cells). Quantitated cell lineage numbers per core were mapped onto three-dimensional stomach maps to demonstrate the distribution of cells in the stomach (left panels). The scale bars represent the quantitated range of positive cells in a core. Representative cores from the body and antrum are shown at the right of each panel with high magnification insets showing the individual cell staining pattern. In the antral core for MUC6 staining in (D), insets show staining for cells with mucous neck cell morphology on the left, and cells with deep antral gland cell morphology on the right.

chief cells and mucous neck cells. We did not strictly quantify surface mucous cell numbers because staining for either MUC5AC or diastase resistant Periodic acid-Schiff was so intense and the cell borders were difficult to discern (data not shown). Online supplementary figure S4A demonstrates that MUC5AC staining surface cells were prominent in cores from both the body and antral regions, but the length of foveolar regions was greater in the antral cores.

We evaluated the presence of proliferating cells using Ki-67 staining (figure $3 \mathrm{~A}$ ). As expected, in the body of the stomach, Ki-67 expressing progenitor cells were located in the upper gland region deep to the foveolar cells. Similarly, in the antrum, Ki-67 expressing cells were present in the mid-gland deep to the foveolar cells. Overall, analysis of the distribution of Ki-67 expressing progenitor cells demonstrated a relatively uniform distribution of proliferative cell numbers throughout the gastric mucosa (figure 4F).
H/K-ATPase staining, as expected, demonstrated large numbers of parietal cells throughout the anatomical body in all three stomachs, with $95 \%$ of parietal cells found within the body mucosa (figures 3B and 4G). However, we also found that all three stomachs showed prominent numbers of parietal cells in groups of glands in the antral region. While the numbers of parietal cells in antral glands represented only $5 \%$ of the total number in the stomach, they were consistently present in the antrum extending towards the pyloric junction (see online supplementary figure S3). The distribution of MIST1 immunoreactive chief cells followed a pattern similar to that seen for parietal cells, with $91 \%$ of cells found in the anatomical body (figures $3 \mathrm{C}$ and $4 \mathrm{H}$ ). However, we again observed chief cells in groups of glands throughout the anatomical antrum.

Identification of mucous neck cells is predicated on the use of antibodies against MUC6 or its companion trefoil protein, TFF2. However, MUC6 and TFF2 are also secreted from the 

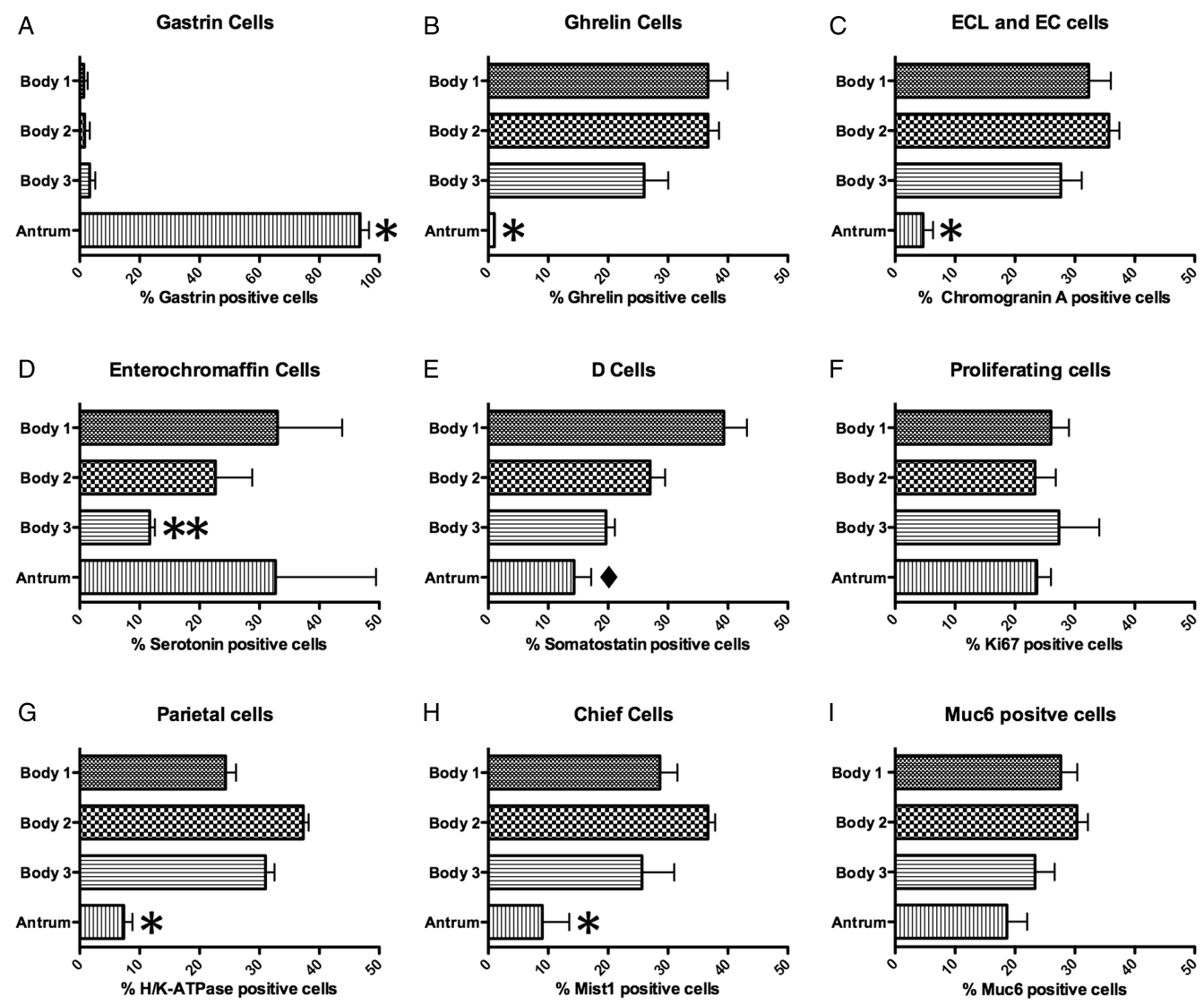

Figure 4 Quantitation of the distribution of cell lineages in the human stomach. To quantitate the distribution of cell lineages within the human stomachs, specimens were divided into three body regions (proximal to distal): body 1 (B1), body 2 (B2), body 3 (B3) and the antrum. The numbers of cells staining for each lineage in each zone were determined as a percentage of the total labelled cells in each stomach specimen. The percentages of labelled cells in each region were compared with ANOVA and Bonferroni's test for significant means. * $p<0.05$ between the antrum and all three body regions; $\wedge p<0.05$ comparing the antrum with B1 and B2; ${ }^{*} p<0.05$ comparing B3 with both B1 and the antrum. All bars represent the mean \pm SEM. ECL and EC cells, enterochromaffin-like and enterochromaffin cells.

deep antral gland mucous cells as well as from Brunner's glands. $^{22} 23$ Thus, as expected, we observed the presence of MUC6 expressing cells throughout the stomach (figures 3D and 4I). In the fundic region, MUC6 immunoreactive cells displayed a small triangular cell morphology and were present in the mid-gland region, all characteristics of mucous neck cells (figure 3D). However, in the antral region, cells with two different morphologies were observed. Some glands showed the morphology of mucous neck cells in the mid-gland regions but others showed the foamy, open ended morphology classically ascribed to deep antral gland mucous cells (figure 3D). Thus together these data suggested that oxyntic type glands were present throughout the human antrum.

\section{The human antral mucosa is assembled from a mixture of 'oxyntic' and 'gastrin' glands}

To analyse in greater detail the structure of glands in the antrum, we performed dual staining for H/K-ATPase and gastrin on arrayed samples of the stomach and paraffin sections of human antrum from six other organ donor specimens. Figure 5 demonstrates that we observed a marked heterogeneity among gland types in the anatomical antrum, with groups of glands containing gastrin cells (figure 5D) interspersed with glands containing parietal cells (figure 5C). Most of the antral cores where gastrin cells were present showed heterogeneity among the glands. In addition, $50 \%$ of glands showed a 'mixed' phenotype containing both gastrin cells and parietal cells (figure 5B). These mixed-type glands contained fewer parietal cells per gland compared with the oxyntic glands in the body, and the parietal cells were generally located deep to the gastrin expressing cells. Within these glands, we occasionally observed cells, which co-stained for both $\mathrm{H} / \mathrm{K}-\mathrm{ATPase}$ and gastrin (see online supplementary figure S5A). These findings suggest that H/K-ATPase and gastrin expressing cells in the antrum might be derived from the same progenitor cell population.

\section{Characterisation of antral gland subtypes}

To examine the relationship of these three gland types in the distal human stomach, we stained 13 serial sections from the human antrum for gastrin and H/K-ATPase along with p120 to outline the lateral membranes of cells (figure $5 \mathrm{E}$ and see online supplementary figure S5B). The images of the stained serial sections were then used to assemble three-dimensional reconstructions of the gland structures (see online supplementary video 10). The reconstruction shows that the gland types, while separated in the deep portions, merged with each other in the upper foveolar regions (see online supplementary figure S5B). Furthermore, foveolar regions often displayed further ramifications. These results suggest that the deep antral glands form with multiple lineage configurations.

As the oxyntic-type glands in the antrum clearly contained only a third the number of parietal cells observed in the oxyntic 

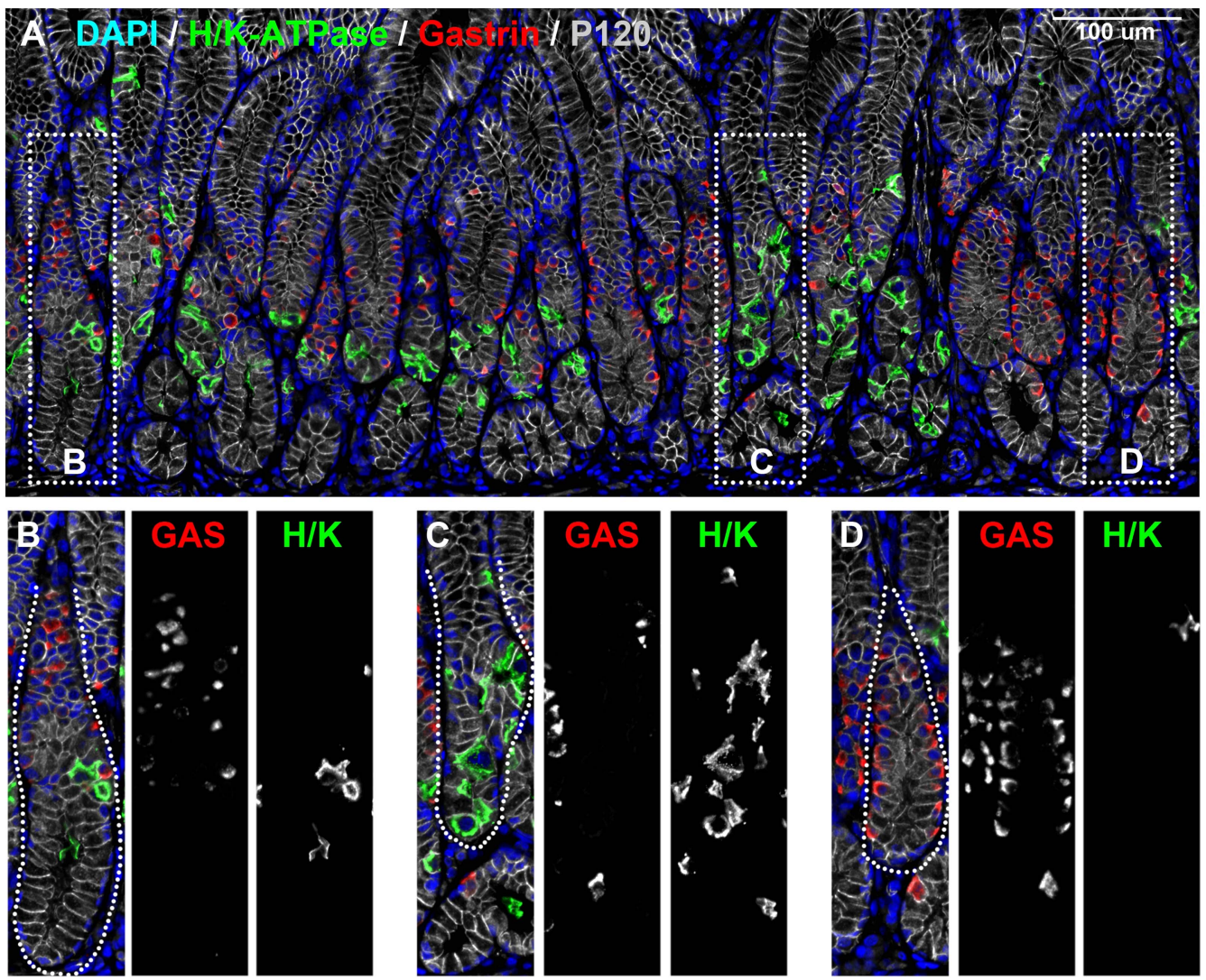

$\mathbf{E}$

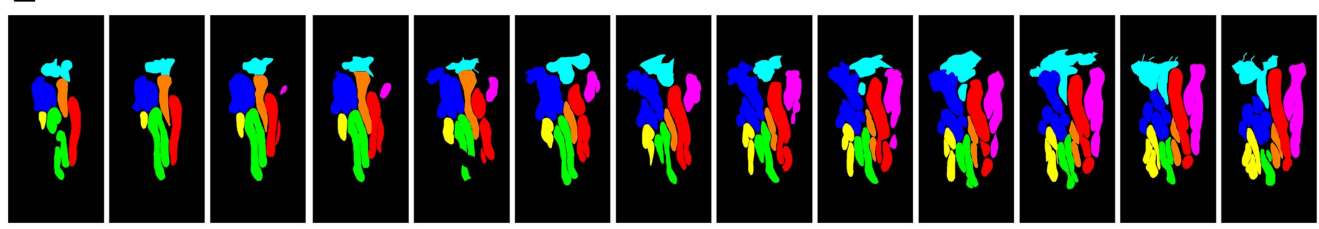

Figure 5 Immunofluorescence staining for parietal cells in human antrum. Paraffin sections of human gastric antrum were immunostained for $\mathrm{H} /$ K-ATPase (H/K) (parietal cells, green) and gastrin (GAS) (G cells, red). P120 (grey scale) immunostaining was used for lateral membrane staining and 4',6-diamidino-2-phenylindole (DAPI) (blue) was used for nuclear staining. Dotted boxes in (A) indicate regions enlarged in (B-D). Three populations of glands were observed in the human antrum: (B) oxyntic glands with parietal cells but not gastrin cells, (C) mixed glands with both parietal cells and gastrin cells and (D) antral-type glands with gastrin cells. Scale bars are as indicated. (E) Tracings of glands in 13 serial sections. Gland morphologies were defined as 'gastrin' or 'mixed' based on triple labelling with antibodies against gastrin (red), H/K-ATPase (green) and p120 (blue). The colour coding for traced gland units was as follows: red, orange and green, antral-type glands lacking parietal cells; pink and yellow, mixed-type glands with both parietal cells and gastrin cells; and light blue and blue, incompletely mapped glands.

glands in the stomach body, we next sought to determine if these glands contained other lineages traditionally observed in oxyntic glands. We utilised the morphology of TFF2 expressing cells to assess the presence of mucous neck cells. Figure 6A demonstrates that parietal cell containing glands also contained mucous neck cells. These cells were clearly distinguishable as small triangular cells compared with the larger basal antral gland-type cells. We also stained for MIST1 expressing chief cells, which are derived from mucous neck cells. MIST1 expressing chief cells were also present in glands with parietal cells, but not in glands with gastrin cells (figure 6B).

Immunohistochemical studies had demonstrated that the antral glands were essentially devoid of ghrelin cells, indicating that there were differences in the antral oxyntic glands. Histamine secreting ECL cells perform a central role in the stimulation of acid secretion by parietal cells. ${ }^{24} \mathrm{~A}$ previous investigation had demonstrated that ECL cells are present throughout the human stomach. ${ }^{19}$ We therefore sought to determine whether the antral glands with parietal cells also contained histamine secreting ECL cells by staining for histadine decarboxylase. Histadine decarboxylase immunoreactive cells were observed throughout the antrum, and dual labelling studies showed that ECL cells were present in all three types of antral glands (see online supplemental figure S6).

Previous investigations in rodents have suggested that antral gland cells express the transcription factor Pdx1, which is responsible for patterning in the distal foregut. ${ }^{10} \mathrm{We}$ therefore stained human antral samples for Pdx1 to determine whether the parietal cells in the antrum also express this antral marker (figure 6C). Pdx1 expressing cells were observed in glands with gastrin cells but not in glands with only parietal cells. Pdx1 co-labelled with gastrin cells in the 'mixed' glands. However, Pdx1 was not observed in the nuclei of H/K-ATPase expressing parietal cells, suggesting that $\mathrm{Pdx} 1$ expressing antral progenitor cells may not give rise to parietal cells in the mixed glands. 

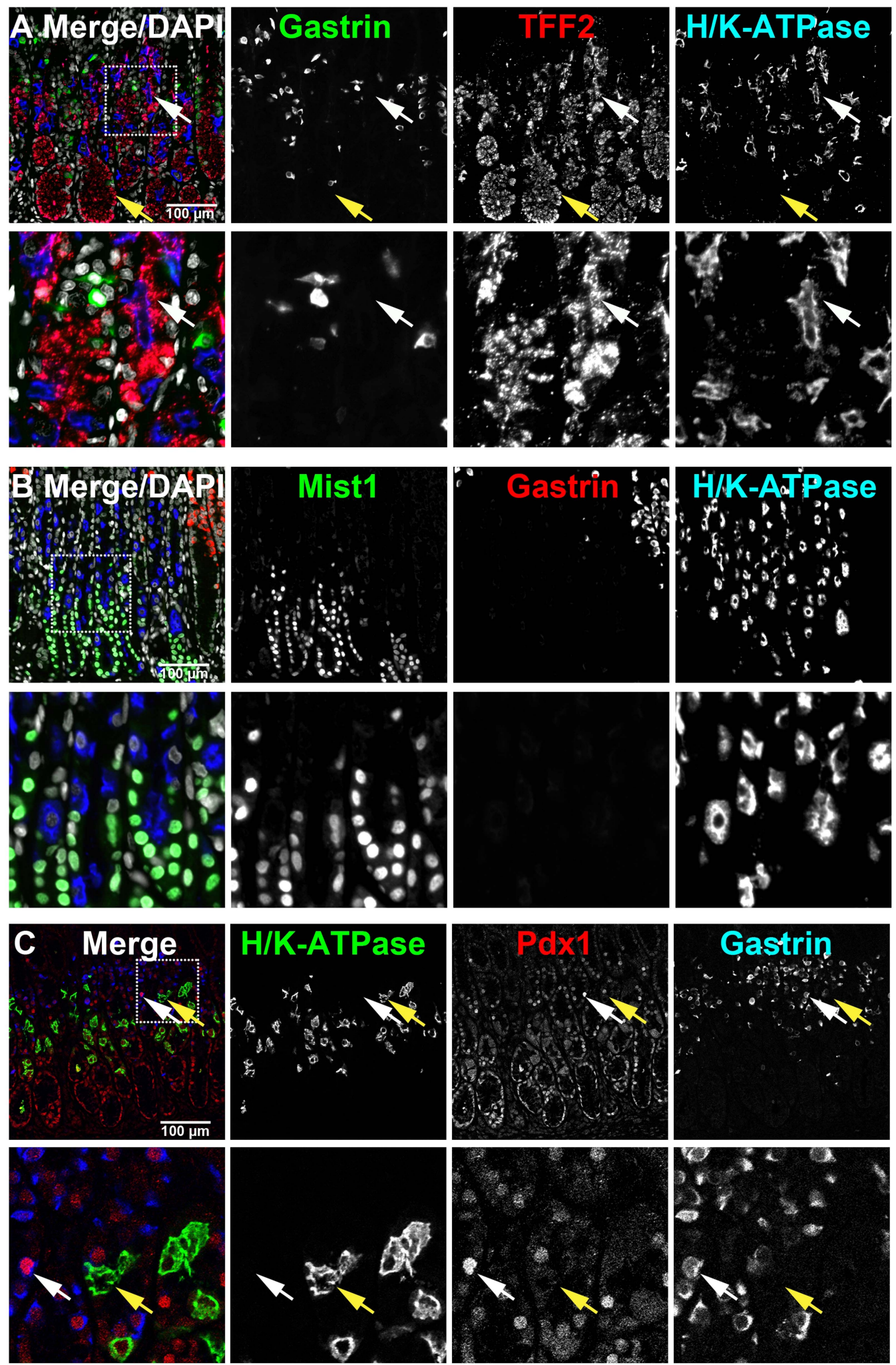

Figure 6 Characterisation of gastric glands in human antrum. Paraffin sections of human gastric antrum were immunostained for trefoil factor 2 (TFF2) (A), MIST1 (B) or pancreatic and duodenal homeobox 1 (Pdx1) (C) with both gastrin and H/K-ATPase. 4',6-Diamidino-2-phenylindole (DAPI) was used for nuclear staining. In (A), the mucous neck cells (white arrows) and the deep antral mucous cells (yellow arrows) were immunostained for TFF2. In (C), gastrin expressing G cells were co-localised with Pdx1 (white arrows), however, H/K-ATPase expressing parietal cells were not co-labelled with Pdx1 (yellow arrows). Dotted boxes depict regions enlarged. Scale bars are as indicated.

Putative gastric stem cells in the human antrum

To understand how the mixture of cell lineages is assembled in antral glands, we next examined the distribution of putative gastric stem cells. In a mouse study, SOX2 expressing stem cells in the stomach were found in both the body and antrum, and the SOX2 expressing stem cells gave rise to both oxyntic and 
antral gland lineages. ${ }^{25}$ We observed SOX2 expressing cells in the antral specimens of the human stomachs. SOX2 expressing cells were widely distributed throughout all three types of glands (see online supplementary figure S7A). Some SOX2 expressing cells were located between parietal cells and gastrin cells, and we observed that $1.95 \%$ of cells were co-positive for both SOX2 and H/K-ATPase and 2.50\% were co-positive for both SOX2 and gastrin (see online supplementary figure S7A, white arrows and see online supplementary figure S7C). We also stained for Ki-67 to assess whether the SOX2 expressing cells were proliferative (see online supplemental figure S7B). We observed that the Ki-67 positive progenitor cells were located adjacent to SOX2 expressing cells. However, SOX2 expressing cells did not co-label with Ki-67. Thus these data suggested that the SOX2 expressing cells may represent a candidate of putative quiescent stem cells in the human antrum, which can give rise to both 'oxyntic' and 'antral' gland lineages.

\section{DISCUSSION}

The present results have defined in detail for the first time the geographic anatomy of cell lineages within the human stomach. The findings here indicate that enteroendocrine cells are not, in general, uniformly distributed in the human stomach. Indeed ghrelin and gastrin are the best markers of the anatomical body and antrum, respectively. A concentration of somatostatin cells was observed in the proximal stomach. Previous studies have noted the enrichment of ghrelin cells in the human body of the stomach. ${ }^{16}$ Other studies have suggested that large numbers of enteroendocrine cells are present in the fetal human stomach before lineages such as parietal cells and chief cells that develop late in gestation. ${ }^{26}$ Thus given the extensive projections that are present for most enteroendocrine cells, ${ }^{27}$ it is tempting to suggest that enteroendocrine cells may be a critical influence for the differentiation of gastric lineages during development as well as during adult life. ${ }^{28} 29$ Alternatively, concentrations and regionalisation of enteroendocrine cells may coordinate local aspects of gastric physiology.

In the present studies, the antrum in the Helicobacter Pylori negative organ donors contained a heterogeneous complement of glands (figure 7), including those classically 'antral' lineages (gastrin cells and deep antral mucous cells), those with oxyntic lineages (parietal cells and chief cells) and glands with mixed lineages (both parietal cells and gastrin cells). These observations contrast with studies in a number of animal species, including rodents, rabbits, pigs, cats and dogs, where the fundic and antral regions segregate glandular mucosa containing the acid secreting parietal cells from the gastrin cell containing antrum. ${ }^{1-5} 7^{30}$ Most textbook interpretations of gastric anatomy in humans have supported this notion, albeit with some acknowledgement that scattered parietal cells can be observed in the human antrum. ${ }^{31-33}$ A number of studies have noted that a transitional zone exists between the body and antrum on both the lesser and greater curvatures in humans as well as in rodents. ${ }^{6} 1234$ This transitional zone contains glands with fewer parietal cells and may vary in length, especially along the greater curvature. ${ }^{12}$ In our present studies, we also observed about a $90 \%$ decrease in parietal cell and chief cell numbers beginning in a region that corresponded to the border between the ghrelin expressing body and the gastrin expressing antrum (figure 4). However, in contrast with previous investigations that noted a transition zone at the border of the body and antrum, we observed parietal cell and chief cell containing glands all the way to the pylorus.
Similar to our present findings, three previous investigations did note significant numbers of parietal cells in the human antrum: in his seminal paper in 1933, Berger noted the presence of parietal cells detected by haematoxylin and eosin staining in the antral region. ${ }^{35}$ Naik et al ${ }^{11}$ found that while gastrin cells clearly defined the anatomical border of the antrum, parietal cells were present in glands in the anatomical antrum. Nevertheless, perhaps the most detailed study was published in 1975 by Tominaga, ${ }^{36}$ who reported parietal cells in the antral mucosa of 116 of 118 subjects. This work suggested that an absence of parietal cells in the human antrum was uncommon, and that the number of parietal cells in the antrum was not affected by gastritis. Another report has commented on the 'unusual' existence of an acidic antrum, which was not amenable to acid reduction with highly selective vagotomy. ${ }^{37}$ Our present findings support the results of these previous investigations and provide further evidence that the parietal cells in the human antral mucosa represent the presence of a variant of oxyntic glands. These antral oxyntic glands have $63 \%$ fewer parietal cells per gland, but also contain both chief cells and mucous neck cells (figure 7). In addition, these glands appear to have a more prominent foveolar mucous cell component. Nevertheless, it is interesting to note that these antral oxyntic glands, unlike their counterparts in the body, do not contain any ghrelin cells. Mixed glands have both parietal cells and gastrin cells, but do not contain chief cells.

Previous investigations in animal models have emphasised developmental regional borders within the gastrointestinal tract that define the boundaries of mucosal lineage derivation. ${ }^{10}$ Thus the antrum and glandular body in rodents contain gastrin or parietal cells, respectively. While transitional glands are present between the rodent body and antrum, ${ }^{6}$ they do separate homogeneous regions of oxyntic glands versus antral mucous glands. The derivation of the antrum in the mouse correlates with the domain in the distal foregut for expression of the regulatory transcription factor Pdx $1 .{ }^{10}$ Glands in the antrum of rodents appear to be derived from Lgr5 expressing stem cells ${ }^{38}$ whereas the glands of the fundic mucosa are not derived from such cell populations. $^{39}$ Our present findings suggest that the human antral mucosa is often made up of a mixture of oxyntic-type and antral-type glands. We have also identified a population of glands with mixed lineages (figure 7). In addition, occasional cells were observed expressing both H/K-ATPase and gastrin (see online supplementary figure S7). These findings indicate that a different paradigm must exist to explain the mixed gland phenotype in humans. The SOX2 expressing stem cells that are considered as marking quiescent stem cell populations are present in the mixed glands in human antrum. Thus in humans, these SOX2 expressing stem cells may be able to give rise to both gastrin cells and parietal cells. Alternatively, mixed glands may contain two distinct sets of progenitor cells necessary for generating either parietal cells or gastrin cells. Nevertheless, in the context of the mixed glands, these progenitors appear to generate not only all of the gastrin gland lineages (surface cells, gastrin cells and deep antral mucous cells), but also parietal cells and ECL cells, without producing other normal oxyntic lineages (mucous neck cells and chief cells) (figure 7). Nevertheless, the presence of other glands that are completely oxyntic or completely antral indicates that specialisation is also possible. Wright et al have previously noted that glands may replicate by fission to yield patches of glands derived from a single founder gland. ${ }^{40}$ As the range of plasticity is not clear, it is not possible to determine whether mixed glands represent the origin of these patches of oxyntic versus antral glands. 
Figure 7 A new model of the heterogeneous types of human antral glands. Analysis of the composition of gastric cells in human antrum indicates the existence of three types of glands: (1) the antral oxyntic gland, (2) the antral mixed gland and (3) the gastrin cell containing antral gland. The oxyntic gland represents the typical gland type present in the body of human stomach (shown on the left) and contains more parietal cells than the antral oxyntic gland and a less prominent foveolar region. ECL cells, enterochromaffin-like cells.
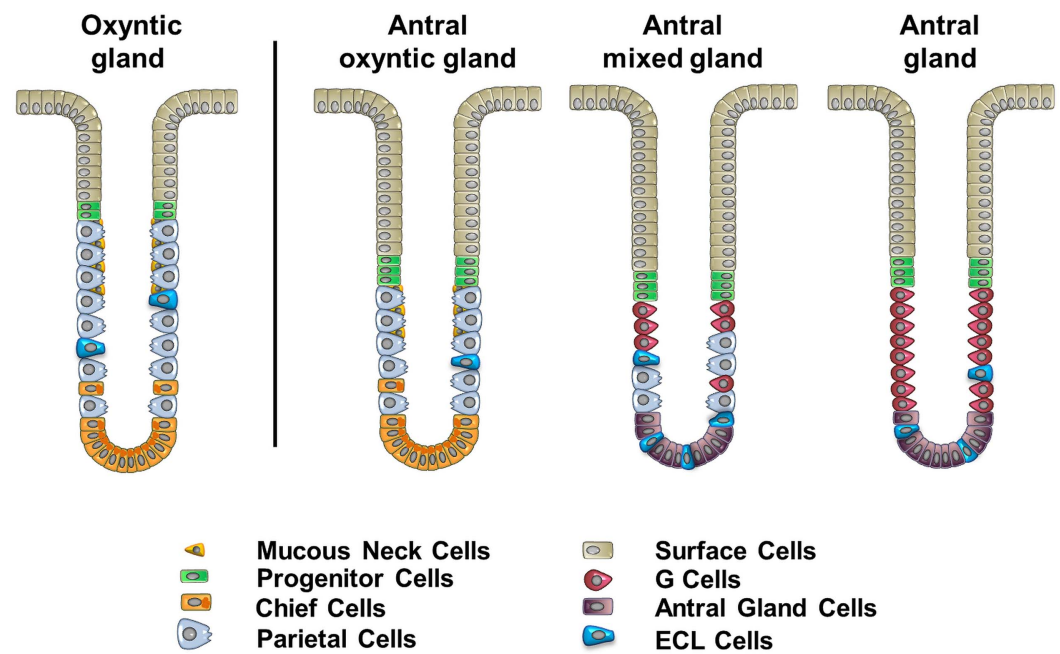

Taken together, these data suggest that there is considerable acid secretory capacity in the antrum from humans. However, it remains unclear why, with the notable exception of the Tominaga paper, ${ }^{36}$ most recent authors have only commented on the presence of a smaller number of parietal cells in the transition zone between the body and the antrum. Tominaga noted no overall difference in the distribution of parietal cells in the antrum of patients with gastritis. ${ }^{36}$ We have examined three complete stomachs and six antral specimens from organ donors, none of whom showed $H$ pylori infection. It is therefore possible that some of the differences may relate to the influence of $H$ pylori on antral gland lineages. However, as Tominaga's observations were made in the 1970s when $H$ pylori infection was extremely common in Japan, this possibility seems less likely. Thus while the inbred rodent strains used in most research may have more uniform patterns of gland geography, humans seem to possess a range of gland derivation patterns in the antrum. It is possible that these differences are related to genetic backgrounds, as examination of human fetal stomachs showed considerable heterogeneity in the distribution of parietal cells in the antrum. ${ }^{41}$ In our own work, we have found no evidence for age related effects on patterns of parietal cell distribution in the antrum. Thus the human population seems to manifest considerable heterogeneity in the presence of mixed and oxyntic glands within the antrum.

In earlier studies in humans and rodents, we have demonstrated that spasmolytic polypeptide expressing metaplasia (SPEM) is associated with local focal changes stemming from parietal cell loss. ${ }^{42} 43$ These changes often involve only single glands, ${ }^{44}$ leading to the suggestion that SPEM reflects a normal reparative response to local damage of gastric glands. Indeed, we did observe instances of single SPEM glands lying within normal mucosa in the donor stomach samples (see online supplementary figure S8). In our previous investigations, we have focused on metaplasia in the fundic region of the stomach, because it was difficult to identify morphologically the presence of SPEM in the antrum where the deep antral gland cells have similar morphology and express similar markers (MUC6 and TFF2) as SPEM cells. Nevertheless, our findings that the human antrum has a mixture of gland types raises the question of the glandular origin of intestinal metaplasia in the human antrum. Previous investigations have noted that gastrin cells are completely absent in glands with intestinal metaplasia in the antrum. ${ }^{45}$ Thus it is possible that intestinal metaplasia (as well as SPEM) might arise from oxyntic glands within the human antrum. This concept would provide a unified explanation for metaplastic processes in the stomach.

In summary, the present investigations demonstrate that a complete examination of the distribution of lineages within the human stomach has revealed complexity or heterogeneity in lineage distribution in the human antrum compared with lower mammalian species. The presence of three discrete types of glands within the human antrum suggests that the pattern of lineage derivation in the distal human stomach is more complicated than that detailed in rodent models. We have also documented regional concentrations of enteroendocrine cells within the stomach. Taken together, these findings indicate that geographic distributions of cell lineages and gland configurations within the human stomach may contribute to key aspects of gastric physiology and pathophysiology.

Acknowledgements This work is dedicated to the memory of Dr John G Forte, who inspired two generations of scientists to understand the physiology of gastric acid secretion. These studies utilised Ariol SL-50 imaging in the VUMC Digital Histology Shared Resource. We thank Dr Al Reynolds for antibodies against p120. We are grateful for the continuing assistance of the staff of Tennessee Donor Services whose efforts at organ procurement made this work possible.

Contributors EC and JTR: designed and performed the studies, analysed the data and drafted the manuscript. BJB, RO and AER: performed the studies. KTN: designed and performed the studies, and analysed the data. CS: designed the studies and analysed the data. JRG: designed the studies, analysed the data and drafted the manuscript.

Funding These studies were supported by a Translational Pilot Grant Award from the Vanderbilt Digestive Disease Research Center (NIH P30 DK058404), R24 DK096527 and R01 DK071590 to JRG. This work was supported by core resources of the Vanderbilt Digestive Disease Center (P30 DK058404) and the VanderbiltIngram Cancer Center through NCI Cancer Center Support grant P30 CA068485, utilising the Translational Pathology Shared Resource.

\section{Competing interests None.}

Ethics approval The study was approved by the institutional review board of Vanderbilt University School of Medicine.

Provenance and peer review Not commissioned; externally peer reviewed.

\section{REFERENCES}

1 Karam SM, Leblond CP. Dynamics of epithelial cells in the corpus of the mouse stomach. I. Identification of proliferative cell types and pinpointing of the stem cells. Anat Rec 1993;236:259-79.

2 Karam SM, Leblond CP. Dynamics of epithelial cells in the corpus of the mouse stomach. II. Outward migration of pit cells. Anat Rec 1993;236:280-96.

3 Karam SM, Leblond CP. Dynamics of epithelial cells in the corpus of the mouse stomach. III. Inward migration of neck cells followed by progressive transformation into zymogenic cells. Anat Rec 1993;236:297-313. 
4 Karam SM. Dynamics of epithelial cells in the corpus of the mouse stomach. IV. Bidirectional migration of parietal cells ending in their gradual degeneration and loss. Anat Rec 1993:236:314-32.

5 Karam SM, Leblond CP. Dynamics of epithelial cells in the corpus of the mouse stomach. V. Behavior of entero-endocrine and caveolated cells: general conclusions of cell kinetics in the oxyntic epithelium. Anat Rec 1993;236:333-40.

6 Lee ER, Trasler J, Dwivedi $S$, et al. Division of the mouse gastric mucosa into zymogenic and mucous regions on the basis of gland features. Am J Anat 1982:164:187-207.

7 Bowie DJ. The distribution of the chief or pepsin-forming cells in the gastric mucosa of the cat. Anat Record 1940;78:9-17.

8 Que J, Luo X, Schwartz RJ, et al. Multiple roles for Sox2 in the developing and adult mouse trachea. Development 2009;136:1899-907.

9 Wells JM, Melton DA. Vertebrate endoderm development. Annu Rev Cell Dev Biol 1999;15:393-410.

10 Larsson LI, Madsen OD, Serup P, et al. Pancreatic-duodenal homeobox 1-role in gastric endocrine patterning. Mech Dev 1996;60:175-84.

11 Naik KS, Lagopoulos M, Primrose JN. Distribution of antral G-cells in relation to the parietal cells of the stomach and anatomical boundaries. Clin Anat 1990;3: $17-24$.

12 Stave $\mathrm{R}$, Brandtzaeg $\mathrm{P}$, Nygaard $\mathrm{K}$, et al. The transitional body-antrum zone in resected human stomachs. Anatomical outline and parietal-cell and gastrin-cell characteristics in peptic ulcer disease. Scand J Gastroenterol 1978;13:685-91.

13 Stave R, Elgjo K, Brandtzaeg P. Quantification of gastrin-producing cells ( $G$ cells) and parietal cells in relation to histopathological alterations in resected stomachs from patients with peptic ulcer disease. Scand J Gastroenterol 1978;13:747-57.

14 Royston CM, Polak J, Bloom SR, et al. G cell population of the gastric antrum, plasma gastrin, and gastric acid secretion in patients with and without duodenal ulcer. Gut 1978;19:689-98.

15 Nielsen HO, Teglbjaerg PS, Hage E. Gastrin and enteroglucagon cells in human antra, with special reference to intestinal metaplasia. Scand J Gastroentero/ Supp/ 1979;54:101-3.

16 Rindi G, Necchi V, Savio A, et al. Characterisation of gastric ghrelin cells in man and other mammals: studies in adult and fetal tissues. Histochem Cell Biol 2002;117:511-19.

17 Stengel A, Tache Y. Yin and Yang-the gastric X/A-like cell as possible dual regulator of food intake. J Neurogastroenterol Motil 2012;18:138-49.

18 Rindi G, Buffa R, Sessa F, et al. Chromogranin A, B and C immunoreactivities of mammalian endocrine cells. Distribution, distinction from costored hormones/ prohormones and relationship with the argyrophil component of secretory granules. Histochemistry 1986:85:19-28.

19 Simonsson M, Eriksson S, Hakanson R, et al. Endocrine cells in the human oxyntic mucosa. A histochemical study. Scand J Gastroenterol 1988;23:1089-99.

20 Ito $\mathrm{H}$, Yokozaki H, Tokumo K, et al. Serotonin-containing EC cells in normal human gastric mucosa and in gastritis. Immunohistochemical, electron microscopic and autoradiographic studies. Virchows Arch A Pathol Anat Histopathol 1986:409:313-23

21 Kasacka I, Lebkowski W, Janiuk I, et al. Immunohistochemical identification and localisation of gastrin and somatostatin in endocrine cells of human pyloric gastric mucosa. Folia Morphol 2012:71:39-44

22 Hanby AM, Poulsom R, Singh S, et al. Spasmolytic polypeptide is a major antral peptide: Distribution of the trefoil peptides human spasmolytic polypeptide and pS2 in the stomach. Gastroenterology 1993;105:1110-16
23 Elia G, Chinery R, Hanby AM, et al. The production and characterization of a new monoclonal antibody to the trefoil peptide human spasmolytic polypeptide. Histochem J 1994;26:644-7.

24 Hersey SJ, Sachs G. Gastric acid secretion. Physiol Rev 1995;75:155-89.

25 Arnold K, Sarkar A, Yram MA, et al. Sox2(+) adult stem and progenitor cells are important for tissue regeneration and survival of mice. Cell Stem Cell 2011:9:317-29.

26 Mitrovic O, Micic M, Radenkovic G, et al. Endocrine cells in human fetal corpus of stomach: appearance, distribution, and density. J Gastroenterol 2012;47:1212-20.

27 Hauso 0, Gustafsson BI, Waldum HL. Long slender cytoplasmic extensions: a common feature of neuroendocrine cells? J Neuroendocrinol 2007;19:739-42.

28 Aikou S, Fukushima $Y$, Ogawa $\mathrm{M}$, et al. Alterations in gastric mucosal lineages before or after acute oxyntic atrophy in gastrin receptor and $\mathrm{H} 2$ histamine receptor-deficient mice. Dig Dis Sci 2009:54:1625-35.

29 Nozaki K, Weis V, Wang TC, et al. Altered gastric chief cell lineage differentiation in histamine-deficient mice. Am J Physio/ Gastrointest Liver Physiol 2009;296:G1211-20.

30 Miyagawa $Y$. The exact distribution of the gastric glands in man and in certain animals. J Anat 1920;55:56-67.

31 Del Valle J, Todisco A. Textbook of gastroenterology. 5th edn. Oxford, UK: Blackwell Publishing, 2009.

32 Boucher IAD, Allan RN, Hodgson HJF, et al. Testbook of gastroenterology. London, UK: Bailliere Tindall, 1984

33 Odze RD, Goldblum JR. Surgical pathology of the GI tract, liver, biliary tract, and pancreas. 2nd edn. Saunders, 2009.

34 Van Zanten SJ, Dixon MF, Lee A. The gastric transitional zones: neglected links between gastroduodenal pathology and helicobacter ecology. Gastroenterology 1999;116:1217-29.

35 Berger EH. The distribution of parietal cells in the stomach: a histopathological study. Am J Anat 1933;54:87-114.

36 Tominaga K. Distribution of parietal cells in the antral mucosa of human stomachs. Gastroenterology 1975;69:1201-7.

37 Simms JM, Bird NC, Johnson AG. An unusual acid antrum. Br J Surg 1985;72:12

38 Barker N, Huch M, Kujala P, et al. Lgr5(+ve) stem cells drive self-renewal in the stomach and build long-lived gastric units in vitro. Cell Stem Cell 2010:6:25-36.

39 Nam KT, O'Neal RL, Coffey RJ, et al. Spasmolytic polypeptide-expressing metaplasia (SPEM) in the gastric oxyntic mucosa does not arise from Lgr5-expressing cells. Gut 2011:61:1678-85.

40 McDonald SA, Greaves LC, Gutierrez-Gonzalez L, et al. Mechanisms of field cancerization in the human stomach: the expansion and spread of mutated gastric stem cells. Gastroenterology 2008;134:500-10.

41 Kelly EJ, Lagopoulos M, Primrose JN. Immunocytochemical localisation of parietal cells and G cells in the developing human stomach. Gut 1993;34:1057-9.

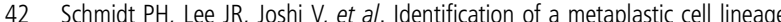
associated with human gastric adenocarcinoma. Lab Invest 1999;79:639-46.

43 Nomura S, Yamaguchi H, Wang TC, et al. Alterations in gastric mucosal lineages induced by acute oxyntic atrophy in wild type and gastrin deficient mice. $\mathrm{Am} \mathrm{J}$ Physiol 2004:288:G362-75.

44 Halldorsdottir AM, Sigurdardottir M, Jonasson JG, et al. Spasmolytic polypeptide expressing metaplasia (SPEM) associated with gastric cancer in Iceland. Dig Dis Sci 2003:48:431-41.

45 Otsuka T, Tsukamoto T, Mizoshita T, et al. Coexistence of gastric- and intestinal-type endocrine cells in gastric and intestinal mixed intestinal metaplasia of the human stomach. Pathol Int 2005;55:170-9. 